\title{
Location-based Software Modeling and Analysis: Tropos-based Approach
}

\author{
Raian Ali, Fabiano Dalpiaz, and Paolo Giorgini \\ University of Trento - DISI, 38100, Povo, Trento, Italy. \\ \{raian.ali, fabiano.dalpiaz, paolo.giorgini\}@disi.unitn.it
}

\begin{abstract}
The continuous growth of interest in mobile applications makes the concept of location essential to design and develop software systems. Location-based software is supposed to be able to monitor the surrounding location and choose accordingly the most appropriate behavior. In this paper, we propose a novel conceptual framework to model and analyze location-based software. We mainly focus on the social facets of locations adopting concepts such as actor, resource, and location-based behavior. Our approach is based on Tropos methodology and allows the analyst to elicit and model software requirements according to the different locations where the software will operate. We propose an extension of Tropos modeling and adapt its process to suit well with the development of location-based software. The proposed framework also includes automated analysis techniques to reason about the relation between location and location-based software.
\end{abstract}

\section{Introduction}

Advances in computing, sensing and communication technology have recently led to the growth of interest in software mobility. Mobility emphasizes several concerns (space, time, personality, society, environment, and so on) often not considered by the traditional desktop systems [1]. Besides computing ubiquity, the 21st century computing [2] is expected to have a core "mental" part: computing systems act on behalf of humans executing tasks without prompting them for and receiving their explicit requests, i.e. computing will realize the concept of agency. Advances in technology do not necessarily imply the easiness of exploiting it, rather more challenges are introduced. Software systems can be given more responsibility, and they can now actively support several decision making processes. Appropriate software development methods and models need to be developed, or adapted, to cope with the new achievable innovative requirements.

Location-based software is characterized by its ability to reason about the surrounding location, including the user, and adapt autonomously a behavior that complies with the location settings. Consequently, we need to model and analyze the variable locations that users can be part of, and define how location influences software. To adopt one behavior, the software needs to reason on what exists and what can be done, basing its choice on user preferences, cost, time, priority, and so on [3]. 
In the area of context modeling, the relation between context and its use is not clearly considered (e.g. [4], [5] and [6]). We believe in the tight complementary relation between the variable behavior (both human and software ones) and context. When the relation between context and its use is omitted, we cannot answer questions like "how do we decide the relevant context?", "why do we need context?" and "how does context influence behavior adaptation?". Modeling context information has not to be a standalone activity, that is context has to be elicited in conjunction with the analysis we do for discovering alternative behaviors. Salifu et al. [7] investigate the use of problem frames to handle variability in context-aware software. In our work, we use goal analysis to elicit requirements without assuming that requirements are already recognized. We also integrate the goal and location models to enable useful automated analysis.

Software variability is a term commonly used to define a software provided with different behaviors, whose variants can be produced guaranteeing low costs, short time, and high quality [8]. Feature modeling is a well known modeling technique exploited by product line engineering to derive a tailored product from a family of possible products [9]. Location-based software is expected to select autonomously among the different alternatives it supports depending on the location settings. Lapouchnian et al. [10] propose techniques to design autonomic software based on an extended goal modeling framework, but the relation with the surrounding location is not focused on. A variant of this approach is proposed by the same authors in [11], where the emphasis is on variability modeling under the requirements engineering perspective, and on the classification of intentional variability when decomposing a goal. In our work, we focus on the variability of location, i.e. the unintentional variability, which influences the applicability and the efficiency of each goal satisfaction alternative.

Goal models, mainly adopted by KAOS [12] and Tropos [13,14] methodologies, represent a paradigmatic shift from object orientation. While goal-oriented analysis is more natural for the early stages of requirement analysis, the objectoriented analysis fits well to the later stages [15]. With goal models, we take a high level goal and start a top-down analysis to discover the more specific subgoals and tasks that are needed to satisfy that goal. Goal model allows for different alternatives to satisfy a goal, but it does not specify where each alternative can be adopted. Alternative behaviors and location variability are complementary. Supporting two alternative behaviors without specifying when to follow each of them rises the question "why do we support two alternatives and not just one?". Conversely, considering location variability without supporting alternative behaviors rises the question "what can we do if the location changes?".

In this paper, we introduce location-based Tropos as a variant of Tropos conceptual modeling framework [13,14], for developing location-based software. We deal with the social level of location, discuss how to model it and how it influences the adaptation of a location-based behavior. We discuss Tropos process for developing location-based software, and then suggest a new variant of it. We introduce three automated analysis on the proposed models to check software against location and vice versa. 
The paper is structured as follows: Section 2 discusses location-based variability and a variety of conceptual modeling challenges introduced by it, and classifies the main features the location-based software in particular has to support. In Section 3, we study Tropos conceptual modeling framework for locationbased software development. In Section 4, we introduce location-based Tropos, proposing modifications on Tropos at both modeling and process levels. In Section 5, we show several kinds of analysis on the new models, and in Section 6, we draw conclusions and present future work.

\section{Location Variability and Location-based Software}

One main concern of software mobility is the ability to perceive the location where the user is, and then tailor a location-based bahavior to achieve user objectives. Location-based software has not only to perceive the technical details of computing environment (communication protocols, network roaming, data interoperability, and so on), but also the social environment the user is part of. The technical level will certainly be the base to handle the low level aspects of software interoperability, related to the machine level. On the other hand, the social level will be the base for tailoring human-oriented behaviors to achieve user goals. In this work, we focus on modeling the social variability of location and how it can be used to derive suitable behaviors for satisfying user goals.

Let us consider a passenger with the goal of buying a ticket in a railway station. Each specific railway station enables different ways to buy tickets (e.g., a passenger can buy a ticket through terminals, e-pay, offices, or through passenger assistance clerks when passenger needs help). Each of these different ways requires specific location properties. For example, buying through terminals requires that a free terminal exists, has one language in common with the passenger, and accept the money or the credit card the passenger has.

In order to satisfy user's needs and goals, location-based software is supposed to be able to select one appropriate behavior according to the location. The behavior has to be compliant with the current state of the location, considering the availability of resources and the existence of other users. Location may be characterized by different dimensions, such as the degree of expertise each user has (in using resources, and communicating with other users), the availability of resources, and the rules that have to be used to coordinate the use of resources, or regulate the interactions between users. In this vision, the conceptual modeling of software system needs to deal with a variety of challenges, such as:

1. Location modeling constructs: we need to find an appropriate set of modeling concepts that can capture efficiently a variable location.

2. Location relevancy: to build a location model, we need a systematic way to decide what has to be modeled, i.e. what is relevant in a location to the target software. E.g. when we model a railway station location, do we need to include passengers current position, or expertise in using PDAs, in the model? and how do we decide that? 
3. Location rules: location, as a system, will impose rules for the interaction among people and for the use of resources. Rules have to be integrated with the location model and modeled using location constructs. E.g. a railway station might impose the rule that only passengers who are foreigners or over a certain age can ask for assistance, and passenger assistant must help even if this implies stoping less priority activity the assistant is involved in.

4. Location-based behavior: to satisfy one of the user objectives, the current location allows a certain set of behaviors. Modeling the relation between the location and the corresponding possible behaviors is essential for locationbased software. E.g. buying a ticket through e-payment can be done only if the station has a network and the passenger is allowed and able to access it.

5. Hierarchial behaviors construction: modeling in a way to avoid "one location, one behavior" enumeration, to exploit commonality of both locations and behaviors fragments, and to enable a hierarchial construction of locationbased behavior. E.g. getting passenger position automatically is a shared objective that needs an automatic positioning system in the station, and this objective is needed to satisfy other objectives like guiding passengers or listing the nearest terminals that in turn is needed for buying a ticket.

6. Location-based behaviors evaluation: based on some payoff functions, each behavior in each location has to be evaluated. We need to model the criteria for evaluating alternative behaviors in variable locations. E.g. when a railway station provides both terminals and e-pay, the software has to decide which one to adopt, and consequently which tasks to do. We need modeling constructs for the criteria on which such kinds of decisions can be taken.

Location-based software is supposed to support mainly five features (hierarchically represented using feature model in Fig. 1):

- Location identification: representing what exists, where the mobile user is, according to a pre-defined location model, i.e. instantiating the location model. E.g. software will receive railway station description and instantiate a railway station model that reflects the current station.

- Location-based behavior adaptation: having an objective, and knowing the current location, the software will reason and select a possible, and even recommended, behavior through which the user objective can be achieved. Behaviors include operational tasks that are done by software, and nonoperational ones the software assists, or simply asks, user to do. To tailor a location-based behavior, the software has to support features like:

- Location-based information processing:

1. Information request: software enables users to request location-based information explicitly, e.g. enabling passengers to ask for the train schedule in the current railway station. Other information requests are implicitly made when location changes, e.g. when train is not in the time, certain information has to be presented to passenger.

2. Relevant information extraction: filtering what is relevant, and composing useful information. E.g. when a train is late, but it is not the passenger train, the warning has not to be shown. Also, when a passenger asks 
how to buy a ticket, and has only cash money that are not accepted by the railway station terminals, the location-based software will exclude terminals from the possible ways of buying a ticket.

3. Information delivery: communicating information to the user in a right way. E.g. notifying the passenger assistant has not to be done by voice message when the assistant is using his/her PDA for a phone call. Also, a demo about using terminals should be interactive, only when the passenger has a good expertise in using PDAs.

- Acting on behalf of user: location-based software will represent the user when interacting with other location actors, both in requesting and answering requests, and in using resources available in a location. E.g. when the passenger asks for a help, the help request will be prepared and sent on behalf of the passenger, including the information needed by the passenger assistant to decide how to accomplish the help.

- Personalization: location-based software will behave differently with different users. Software considers user personality as one location mobility dimension. E.g. when both wireless and wired connections are available in a railway station, and the passenger prefers reliable connection, the software will lead passenger to wired connection terminal, and when passenger wants more easy connection, the software will configure wireless one.

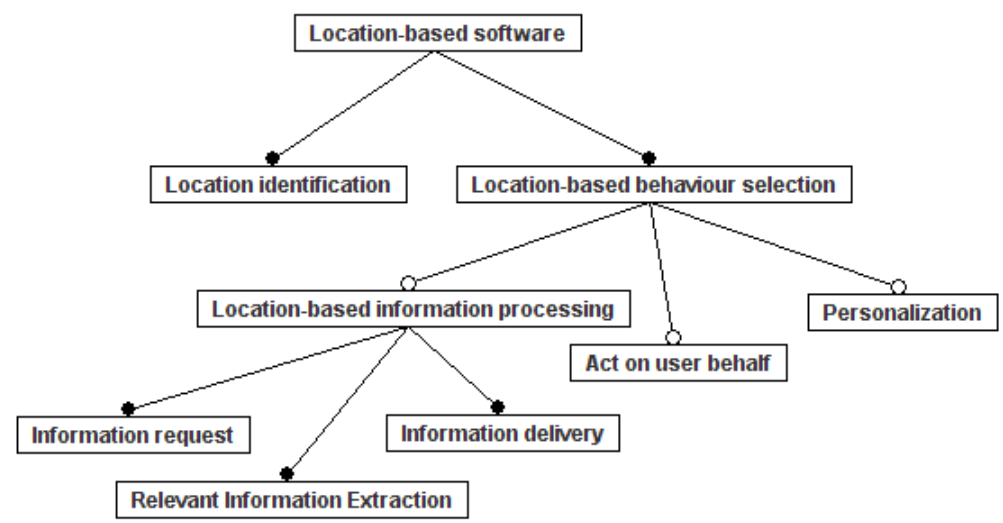

Fig. 1. Feature model for location-based Software

\section{Tropos for Location-based Software}

Our approach is based on Tropos methodology [13,14], which offers an agentoriented conceptual framework for modeling both the social environment and the system-to-be. Tropos starts its software development life cycle with the early requirements phase. In this phase, the organization (location at the social level) 
is modeled as a set of actors that strategically depend on each other for satisfying their objectives, then the rationale of satisfying each actor own objectives is modeled. If we take the railway station scenario, the strategic dependency between railway station actors with respect to the goal Ticket is Issued will be as shown in Fig. 2. Tropos early requirement fits well to project the social structure of the location at a higher level as a set of actors and resources. Taking into consideration a variable location, this phase will not be sufficient enough and we will need to adapt it to deal with points such as:

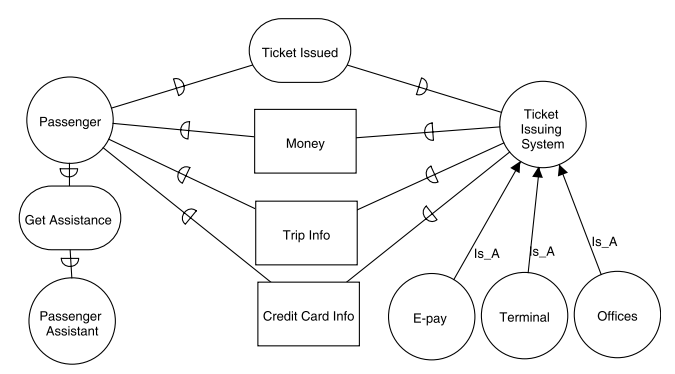

Fig. 2. A strategic dependency model for the railway station scenario.

1. Tropos modeling supposes the existence of all modeled actors (terminal, epay, offices, passenger assistant), and this assumption will not hold when we consider a variable location, i.e. location structure is not static.

2. Tropos modeling has to consider actors and resources profiles to deal with several location modeling difficulties:

(a) Tropos modeling is not able to differentiate between availability levels of actors and resources. In such modeling, all railway station terminals are equally available, but it is more useful to consider a terminal, that is close to the passenger, more available than a terminal which is far away.

(b) Dependencies between actors are not required or achievable in every location, and we can not specify that using the rigid form of describing actors and resources in Tropos modeling. Credit card info can be required when Ticket Issuing System enables payment through credit cards, and when the passenger's credit card is compatible with the supported ones.

(c) When more than one actor is available to satisfy one objective, there is no way to differentiate between them, and then to choose the best. If we consider Terminal and E-Pay as two Ticket Issuing Systems without considering their profiles and matching them with passenger profile, these two ticket issuing systems can not be differentiated.

3. Tropos proceeds, in the next step of early requirements phase, to analyze the rationale of Ticket Issuing System to satisfy Ticket is Issued goal, and that is not what we always need. Ticket Issuing System already exists, and we do not need to develop a software for it, rather for Passenger to deal with this already functioning system. 
In Tropos late requirements phase, the system-to-be is introduced as a new actor that takes some responsibilities, already identified in the first phase, and provides an automated solution. The rational of the system-to-be actor is represented by a goal model, starting with a high level goal and finding alternative sets of behaviors that lead to the satisfaction of that goal. Considering location-based software, the rationale of the system-to-be actor is to find suitable behavior for each possible location. In our railway station scenario, the developed locationbased software will be for passengers, and passenger assistants as mobile actors. It will work as an automated location expert that operates on the user's computing device, and knows both its user and its location social structure.

In a way different from Tropos late requirements, the system-to-be actor is not necessarily assigned an objective that is recognized in the first phase, and is mainly developed to assist users in the already functioning system that is modeled in the first phase. In our example, two system-to-be actors need to be introduced, one for passenger and another for passengers assistant. The rationale of these two location-based software actors is partially shown in Fig. 3. On this goal-oriented rationale model, that represents well the alternative behaviors of location-based software, we can also highlight several remarks:

1. The system-to-be has, in particular, two characteristics:

(a) It is naturally decentralized, that is a location-based software will be assigned for each mobile actor that might also deal with another locationbased software assigned to other actors. In our example, we need two location-based software actors, one for Passengers, and another for Passenger Assistants.

(b) The responsibilities given to the system-to-be actors fall into the categories we have listed in Section 2, and the rationale analysis concerns how to assist the mobile users in an already functioning system. For example, passengers location-based software will choose the way that fits to them and to the station when they need to buy tickets, and it will interact with passengers assistants on behalf of the passengers for a help.

2. Tropos goal analysis supports different alternatives to satisfy the high level goals. What we need is a kind of location-based goal analysis, that adds location properties to each alternative specifying where it can be adopted. For example, in Tropos goal analysis shown in Fig.3, we do not specify where each of the possible alternatives for having a ticket can be adopted.

3. The contribution to softgoals can be location-based, and is not always static. The relation between the contribution and the location is omitted in the current Tropos goal model. For example, the goal Wireless Connection contributes better to the softgoal Reliable Connection when the passenger is close to wireless network access points, than it does when user is far from it.

4. The autonomous selection amongst alternatives, when more than one are available, needs to be specified based on some criteria. For example, in a railway station where offices are opened, terminals are available, and passenger has the ability to adopt each of these alternatives, we need to specify the decision to be taken. 


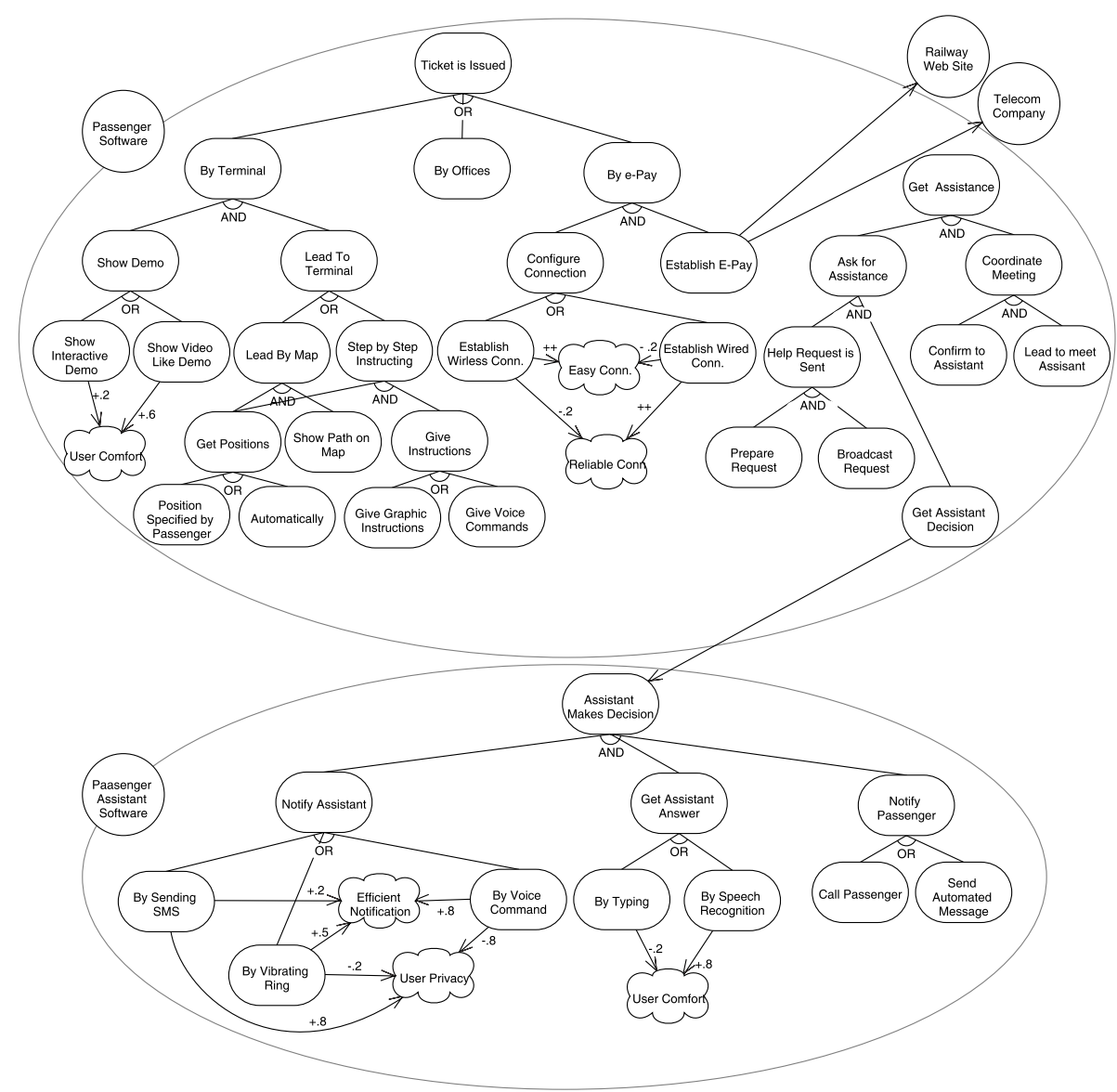

Fig. 3. System-to-be actors goal model for the railway station scenario.

\section{Location-based Tropos}

In the previous section we have addressed the potential and the limitation of Tropos with regards to location-based software development. Early requirements conceptualization, that concerns modeling location, is not sufficient enough to model variable location and needs mainly to consider actors and resources profiles. We have shown the system-to-be, introduced in the late requirements, as a set of location-based software actors that assist mobile actors to satisfy their needs in a location. We have also addressed the gap between Tropos goal-oriented rationale and location, since we need mainly to associate between the goal satisfaction alternatives and the locations where they can be adopted. 
When the analyst builds the goal model shown in Fig. 3, a specific assumption about the location, where each of the alternatives can be adopted, could be thought about but was not explicitly represented in the model. Here we discuss five variation points on Tropos goal model that might need location properties to take location-based decision:

1. Location-based Or-decomposition: Or-decomposition is the basic variability construct; in current Tropos the choice of a specific Or-alternative is left to the actor intention, without considering location properties that can inhibit some alternatives. E.g. the alternative By Terminal can be adopted when a terminal is free, has one language in common with the passenger, and supports the cash money -in both of the type (coins, papers) and the currencyor one credit card the passenger has. The alternative E-Pay can be adopted when there is a wireless network in the railway station and the passenger's PDA supports $\mathrm{WiFi}$, or when there is a wired network with a cable-based connection terminals and the passenger's PDA has cable connectivity.

2. Location-based contribution to softgoals: the value of contributions to softgoals can vary from one location to another. E.g. the goal Interactive Demo contributes positively to the softgoal User Comfort when the user has good expertise in using PDAs, and the used PDA has a touch screen, while the contribution is negative in the opposite case. Also, the goal Wireless Connection contribution to the softgoal Reliable Connection depends on the distance between passenger and WiFi access point to which passenger is connected.

3. Location-based dependency: in some locations, an actor might be unable to satisfy a goal using its own alternatives. In such case, the actor might delegate this goal to another actor that is able to satisfy it. E.g. delegation of the goal Establish E-Pay to the actor Railway Website can be done when that web site enables e-payment using one credit card in common with user's credit cards, and has a mobile device version.

4. Location-based goal activation: an actor, and depending on the location settings, might find necessary or possible triggering (or stopping) the desire of satisfying a goal. E.g. the goal Assistant Makes Decision is activated when the assistant is not doing any particular activity, has one language in common with the requesting passenger, and close to that passenger.

5. Location-based And-decomposition: a sub-goal might (or might not) be needed in a certain location, that is some sub-goals are not always mandatory to fulfill the top-level goal in And-decomposition. E.g. The goal Show Demo has to be satisfied when the passenger is not familiar with using terminals.

The goal analysis of location-based Tropos associates location properties to each location-based variation point. In addition, this analysis helps to refine the initial location model represented in the first phase. If we consider the location properties in the above examples, we can identify how the location model of Fig. 2 can be refined. The resulted location model of the railway station scenario, with respect to the location properties given in the examples above, is shown in Fig. 4. This model adds mainly actors and resources profiles, and also introduces new resources and actors that can influence tailoring location-based behavior. 


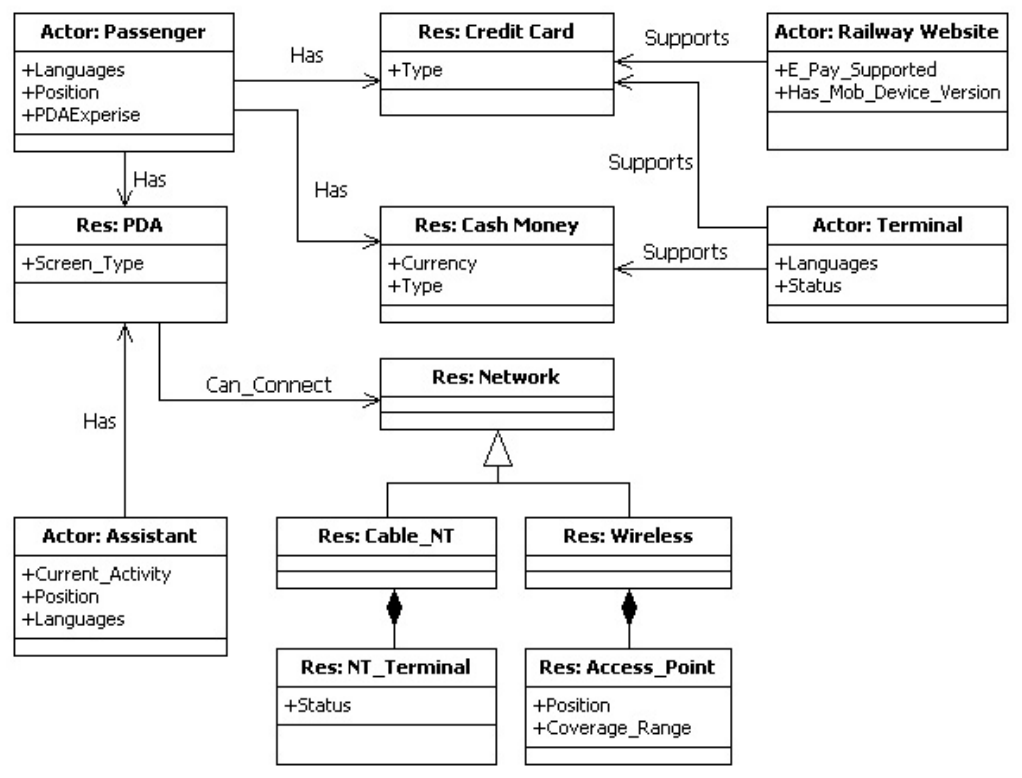

Fig. 4. Location model for the railway station scenario

There are two top-level classes in the location model: actors and resources (Res in the figure). The actor Passenger is characterized by some attributes: spoken Languages (we put it as a single attribute to simplify the diagram), Position in the railway station, and Expertise in using PDAs. The passenger might have three relevant resources: PDA, Credit Card, and Cash Money. The resource PDA is characterized by an attribute Screen_Type, defining if the PDA has a touch screen or not, and it has a Can_Connect association to the Network it can connect to. A network can be specialized into Cable_NT and Wireless. Cable_NT stands for wired networks, and it is composed of a set of network terminals (NT_Terminals), characterized by a Status that can be free, busy, under maintenance, out of service, and so on. Wireless network is composed of several wireless access points (Access_Point); an Access_Point has the attributes Position and Coverage_Range, used together to compute if a customer is covered by an access point signal. The actor Assistant has a Current_Activity he/she is performing, a Position in the railway station, and spoken Languages. The assistant's relevant resources include only the assistant's used PDA. The actor Railway Website has the attributes E_Pay_Supported, to indicate if e-payments are supported, and Has_Mob_Device_Version, set to true when the website can be browsed by PDAs. The Credit_Card resource class represents the types of credit cards passenger might use, and terminals and railway station website might support. The actor Terminal might support multiple Languages, be in a variable Status, and support Credit Card or Cash Money payment. 
We describe now our proposed location-based Tropos process that leads the production of our proposed models. We start by (i) modeling the social structure of a location class, before introducing the system to-be, using a strategic dependency diagram. In this step, we identify roughly the main location actors and the strategic dependencies between them. Then (ii), this diagram is examined to determine a set of mobile actors, i.e. actors who need location-based software to assist them in the considered class of locations. The next step is to (iii) assign a system-to-be actor to each mobile actor, and to model the rationale of these system-to-be actors, using goal analysis. While doing the goal analysis, system analyst (iv) decides those location-based variation points, and specifies the location properties at each of them to help selecting between alternatives. Location properties refine the location model, that consists initially of the actors and resources recognized in the first step. System analyst (v) will extract new location model constructs (actors or resources properties and relations, new resources or actors) that each location property at each location-based variation point might contain, and keep updating the location model.

By following our proposed location-based Tropos process, we will have three models: the first is the classical Tropos strategic dependencies model, the second represents the location-based rationale of the system-to-be actors (Fig. 3 associated with location properties at the location-based variation points), and the third is the elicited location model (the model of Fig. 4). The metamodel of our proposed extension of Tropos modeling is shown in Fig.5.

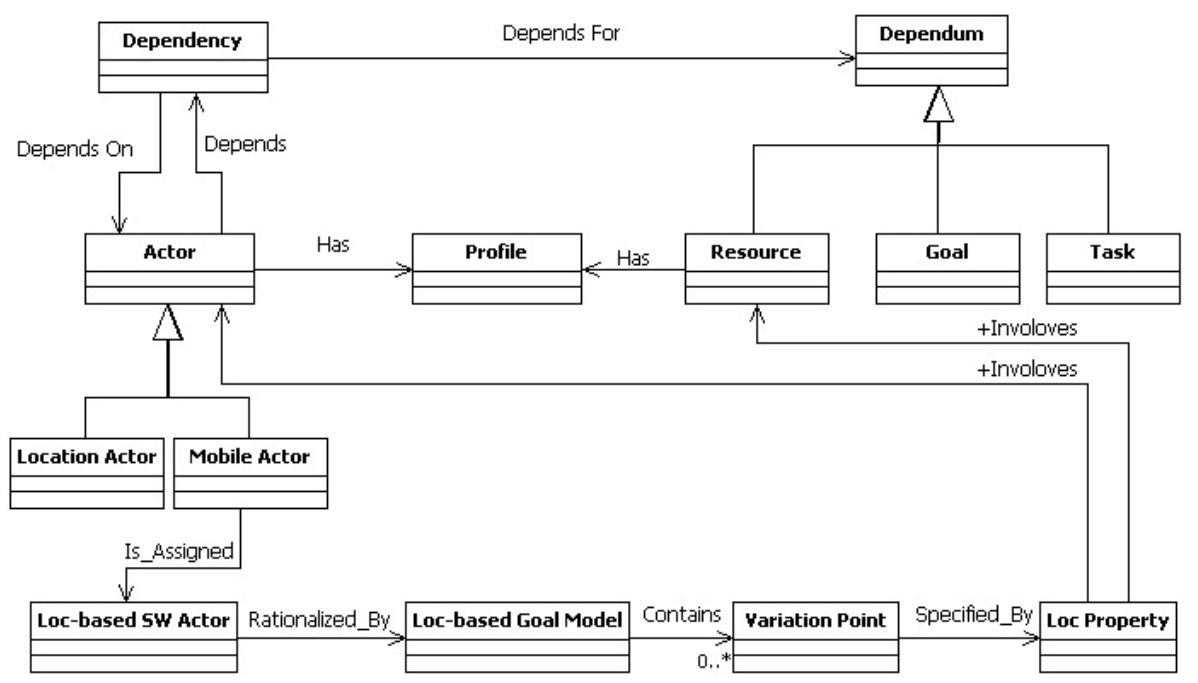

Fig. 5. Metamodel shows the proposed extension of Tropos 


\section{Reasoning on Location-based Models}

We propose various types of analysis for examining location-based software against a specific location, and vice versa. A preliminary step consists of evaluating the validity of location properties at the variation points of the goal model on the current location instance. This step can be done automatically using an automated solver after formalizing the location and location-based goal models. In [16], we used class diagram to represent location, and we formalized it besides the location properties using Datalog $\neg$. We used DLV solver to do the reasoning. Here we discuss several kinds of automated analysis on our proposed models:

- Location-based goal satisfiability: this kind of analysis is aimed to verify if a goal is achievable through one alternative in the current location instance. The analysis can be performed using the goal reasoning algorithm proposed by Giorgini et al. [17] on the goal model restricted by the evaluation of the location properties. A strategy for evaluating satisfiability follows a topdown approach: starting from a top-level goal, we should check that all (at least one) sub-goals in and- (or-) decompositions can be achieved, or that the top-level goal can be achieved via a makes $(+1.0)$ contribution from an achievable goal. For example, in a railway station where there is no positioning system, offices are closed because of vacation, there is a kind of network compatible with the passenger's PDA connectivity, and the railway company website supports one of the passenger's credit cards for e-pay, the algorithm will mark the root goal "Ticket is Issued" as a satisfiable goal. The algorithm finds the alternative E-Pay satisfiable, because of the satisfiability of its two And-decomposition subgoals. The alternative By Terminal can not be satisfied due to the absence of any positioning system, and therefore the unsatisfiability of its and-decomposition subgoal Lead to Terminal that can not be satisfied by any of its alternatives in its turn. The alternative $B y$ Offices can not be adopted, because it requires a location property Offices are working, to be satisfied.

- Location properties satisfiability: this analysis checks if the current location structure is compliant with the software goals. It is exploited to identify what is missing in a particular location where some top-level goals have been identified as unsatisfiable by location-based goal satisfiability analysis. When a goal can not be satisfied, the analysis will identify the denying conditions and suggest ways for solving the problem. For example, in a railway station while passengers have PDAs with only wired connectivity feature, while railway station does not provide cable-based connection terminals, the previous analysis will mark Configure Connection as unsatisfiable goal. The reason is that location properties on each of the two connection modalities, wireless and wired, are not satisfied. Location properties satisfiability will reason on what is needed to satisfy the Configure Connection goal, i.e. what is needed to satisfy location properties on its alternative behaviors. 
- Preferences analysis: this type of analysis requires the specification of preferences over alternatives. As shown in [18], preferences can be specified using softgoals. This analysis is useful in cases like:

- When some locations allow for several alternatives to satisfy a goal: the selection will be based on the contributions (possibly location-based) to the preferred softgoals. For example, in a railway station where both Wireless Connection and Wired Connection can be satisfied, locationbased software will adopt the one preferred by its users. User preferences can be specified over softgoals: when user gives more importance to Reliable Connection than Easy Connection, the Wired Connection alternative will be adopted, while Wireless Connection is adopted when user cares Easy Connection more than Reliable Connection.

- When a certain location does not allow for any alternative to satisfy a goal: the location properties satisfiability might provide several proposals about the needed location modifications. The adopted modifications are those that lead to satisfy more the preferences expressed over softgoals. For example, in one railway station where Configure Connection can not be satisfied due to the absence of wireless network, or cable based terminals, the railway adminstration has to decide between establishing wireless or wired network. When the railway station adminstration cares more Reliable Connection, a wired network terminals has to be installed over the station, while wireless access points will be installed when Easy Connection is more preferred.

\section{Conclusions and Future Work}

In this paper, we have shown the particularity and importance of modeling location variability for location-based software, and addressed some challenges the conceptual modeling faces with this regards. We classified several features location-based software in particular has to support. To develop location-based software we relied on Tropos methodology, and have shown its potential and limitation for developing such software. We have suggested to modify the conceptualization and the process of Tropos to fit well with location-based software development. We have shown three kinds of automated analysis on our proposed location-based models. In this work, we have considered the social level of a location class as a set of profiled actors and resources; our future work will be towards refining this modeling by finding a set of common concepts that can construct more specifically actors and resources profiles and relations. Consequently, we will also need a formal language that is expressive enough to represent the location-based models, and practical for the needed automated analysis.

\section{Acknowledgement}

This work has been partially funded by EU Commission, through the SERENITY project, by MIUR, through the MEnSA project (PRIN 2006), and by the Provincial Authority of Trentino, through the STAMPS project. 


\section{References}

1. Krogstie, J., Lyytinen, K., Opdahl, A., Pernici, B., Siau, K., Smolander, K.: Research areas and challenges for mobile information systems. International Journal of Mobile Communications 2(3) (2004) 220-234

2. Weiser, M.: The Computer for the Twenty-First Century. Scientific American 265(3) (1991) 94-104

3. Ali, R., Dalpiaz, F., Giorgini, P.: Location-based variability for mobile information systems. In: Proc. 20th Intl. Conference on Advanced Information Systems Engineering (CAiSE'08), LNCS 5074, Springer. (2008) 575-578

4. Yau, S.S., Liu, J.: Hierarchical situation modeling and reasoning for pervasive computing. In: Proc. Fourth IEEE Workshop on Software Technologies for Future Embedded and Ubiquitous Systems (SEUS '06). (2006) 5-10

5. Henricksen, K., Indulska, J.: A software engineering framework for context-aware pervasive computing. In: Proc. Second IEEE Intl. Conference on Pervasive Computing and Communications (PerCom'04). (2004) 77

6. Wang, X.H., Zhang, D.Q., Gu, T., Pung, H.K.: Ontology based context modeling and reasoning using owl. In: Proc. Second IEEE Annual Conference on Pervasive Computing and Communications Workshops. (2004) 18-22

7. Salifu, M., Yu, Y., Nuseibeh, B.: Specifying monitoring and switching problems in context. In: Proc. 15th Intl. Conference on Requirements Engineering (RE'07). (2007) 211-220

8. Pohl, K., Böckle, G., van der Linden, F.: Software Product Line Engineering: Foundations, Principles, and Techniques. Springer (2005)

9. Kang, K.C., Kim, S., Lee, J., Kim, K., Shin, E., Huh, M.: Form: A feature-oriented reuse method with domain-specific reference architectures. Ann. Softw. Eng. 5 (1998) 143-168

10. Lapouchnian, A., Yu, Y., Liaskos, S., Mylopoulos, J.: Requirements-driven design of autonomic application software. In: Proc. 2006 conference of the Center for Advanced Studies on Collaborative research (CASCON '06), ACM (2006) 7

11. Liaskos, S., Lapouchnian, A., Yu, Y., Yu, E., Mylopoulos, J.: On goal-based variability acquisition and analysis. In: Proc. 14th IEEE Intl. Requirements Engineering Conference (RE'06). (2006) 76-85

12. Dardenne, A., van Lamsweerde, A., Fickas, S.: Goal-directed requirements acquisition. Sci. Comput. Program. 20(1-2) (1993) 3-50

13. Bresciani, P., Perini, A., Giorgini, P., Giunchiglia, F., Mylopoulos, J.: Tropos: An agent-oriented software development methodology. Autonomous Agents and Multi-Agent Systems 8(3) (2004) 203-236

14. Yu, E.: Modelling strategic relationships for process reengineering. Ph.D. Thesis, University of Toronto (1995)

15. Mylopoulos, J., Chung, L., Yu, E.: From object-oriented to goal-oriented requirements analysis. Commun. ACM 42(1) (1999) 31-37

16. Ali, R., Dalpiaz, F., Giorgini, P.: Modeling and analyzing variability for mobile information systems. In: Proc. Intl. Conf. on Computational Science and its Applications (ICCSA'08), UWSI'08 Workshop, LNCS 5073, Sprniger (2008) 291-306

17. Giorgini, P., Mylopoulos, J., Nicchiarelli, E., Sebastiani, R.: Reasoning with goal models. In: Proc. 21st Intl. Conf. on Conceptual Modeling (ER'02). (2002) 167-181

18. Liaskos, S., McIlraith, S., Mylopoulos, J.: Representing and reasoning with preference requirements using goals. Technical report, Dept. of Computer Science, University of Toronto (2006) ftp://ftp.cs.toronto.edu/pub/reports/csrg/542. 